\title{
Paguettes \& bastries: Novice French learners show shifts in native phoneme boundaries ${ }^{1}$
}

Marisa Tice (Stanford University) \& Melinda Woodley (UC Berkeley)

The idea that second language (L2) learners' phonological categories are tightly linked to their native language categories is not a new one (Laeufer, 1996). Typically, the role of L2 phonology has been seen as subordinate to native (L1) phonology, preventing L2 learners from attaining native-like perception (e.g., English /r/ vs. /l/ for Japanese speakers; Bradlow et al., 1999). Transfer effects from the L1 to the L2 also seem to impede the production of similar but non-identical L2 phones (Flege, 1987).

In the present study, we find that, though L1 and L2 categories are tightly linked in early L2 acquisition, the relationship between them is not strictly one-way. Our findings demonstrate interference from the $\mathrm{L} 2$ on the $\mathrm{L} 1$ in both a phoneme categorization task and an auditory semantic priming experiment, supporting recent work on cross-language effects (e.g. Chang, 2010; Flege, Schirru, \& MacKay, 2003) and underscoring the continued malleability of L1 phonological categories well into adulthood.

Chang (2010) demonstrated that L2-to-L1 transfer effects occur much sooner after the onset of L2 acquisition than was previously thought. Over the course of a 6-week Korean immersion course, his L1 English participants showed significant changes in their production of English stops due to their exposure to L2 Korean. The present study also focuses on L2-to-L1 transfer effects during the first few weeks of language learning. We hoped to learn whether the shifts in L1 speech production observed by Chang would be mirrored in L1 speech perception, and in a future extension of the present work, whether our group of novice learners also showed comparable shifts in L1 production. Assuming that exposure to L2 French would result in a lower VOT category boundary for English stops, we chose two tests of perception: in the first, we directly tested category boundary shift with a phoneme categorization task. In the second, we tested the interaction of category boundary shifts and semantic priming (i.e., does "bastry" [peIstii] (with French-like initial VOT) become a better prime for "chef" with L2 exposure to French?) We find in both cases that participants showed L2-to-L1 transfer effects on their VOT perception in English within the same short time frame that Chang (2010) found for production.

\section{Methods}

Our Learner group consisted of five native American English speakers who had extremely little or no previous exposure to French. One of the five was fluent in another language (Spanish), but the others were either monolingual or had limited language skill with another L2 (Spanish, Latin). All five had enrolled in an intensive beginning French language course for the summer of 2011 in Paris, France and began their participation in our study near the start of their summer coursework. Our Control group consisted of nine native American English speaking undergraduates in the United States who had limited language skill with an L2 and who were not currently enrolled in any language courses.

Each group participated in weekly perception, production, and non-linguistic cognitive tasks for four to six weeks. The Learner group participated in perception and production tasks in both French and English, while the Control group only participated in the English tasks. We report here on the results of our two perception studies.

\footnotetext{
${ }^{1}$ Presented January $7^{\text {th }}, 2012$ at the $86^{\text {th }}$ Annual Meeting of the Linguistic Society of America in Portland, OR.
} 


\section{Phoneme Categorization}

In the phoneme categorization task, participants identified CV syllables along a voice onset time (VOT) continuum (ba-pa, da-ta, and ga-ka). Each continuum contained 12 steps from $-85 \mathrm{~ms}$ to $90 \mathrm{~ms}$ VOT, copy-synthesized based on one prevoiced and one aspirated token. After hearing a CV syllable, participants identified the sound they heard by choosing between two orthographically-presented words that formed a voiced-voiceless minimal pair (e.g., on /ba/-/pa/ trials, "bop" vs. "pop"; /da/-/ta/, "talk" vs. "dock"; /ga/-/ka/ trials, "con" vs. "gone"). Each token from the three stop continua was presented two times (72 tokens total) in randomized order on each of the six testing sessions. Participants were told that they were going to hear sounds from "English" and make decisions about them. The task took about 2 minutes and was given in English and French (same stimuli, different orthographically-represented choices), but here we report only the results for English.

\section{Semantic Priming}

Previous work has demonstrated a phonetic "best fit" effect for auditory semantic priming, such that $\left[\mathrm{k}^{\mathrm{h}} \mathrm{aet}\right]$ is a better prime for "dog" than [kaet] - canonical VOTs activate word representations better than non-canonical ones (Andruski et al., 1994). We hypothesized that exposure to short-lag VOT voiceless stops in French (and French-accented English) would cause [kaet] to become a better prime if learners' English phoneme boundaries shift toward French VOT norms.

Test items were either lexically voiceless-initial (P-type: "peace") or voiced-initial (Btype: "beef") words for which a change in voicing resulted in a nonword ("beace", "peef"). The initial stops were distributed evenly among bilabial, alveolar, and velar places of articulation. Each item was phonetically manipulated to have 0,20 , or $80 \mathrm{~ms}$ of VOT. Items with 0 ms VOT solidly fall within the English voiced category, while items with $80 \mathrm{~ms}$ fall into the voiceless category. Items with $20 \mathrm{~ms}$ VOT were expected to be ambiguous for native English speakers, and most susceptible to a shift in voiced-voiceless boundaries. Each prime-target pair ("peace" "war") was preceded by 1-3 random lexical items to obscure the prime-target relationship and encourage attention to the task. Participants judged whether the target item (the last word) was a "word" or "nonword" of English. Half of the items were nonwords, and the total set included 108 filler trials and 108 test trials, distributed evenly among the three places of articulation and the P-type vs. B-type words. Participants took a short break after the first and second block of 72 trials. The task lasted 12 minutes and was only given in English since French learners with two weeks of L2 exposure have extremely limited vocabularies and would not show significant priming effects.

\section{Results and Discussion}

A small perceptual shift took place over the 6-week testing period for the Learner group. No similar shifts surfaced in the Control group. Overall, the learners show a lower voicedvoiceless boundary than the controls. The average crossover step for the learners was $6.3(\sim 30$ $\mathrm{ms})$ - one full step lower than the controls at $7.3(\sim 35 \mathrm{~ms})$. The Control group showed no change over the six sessions, but the learner group shifted towards an even lower boundary during weeks 3 and 4, shifting back to their early boundary at weeks 5 and 6 . Their overall lower category boundary and downward shift in weeks 3 and 4 are consistent with a shift toward the French voiced-voiceless boundary. The greatest shift appears during the same period that Chang (2010) reported significant L2-related changes in the English speakers' VOT (week 3). 


\section{Control group}

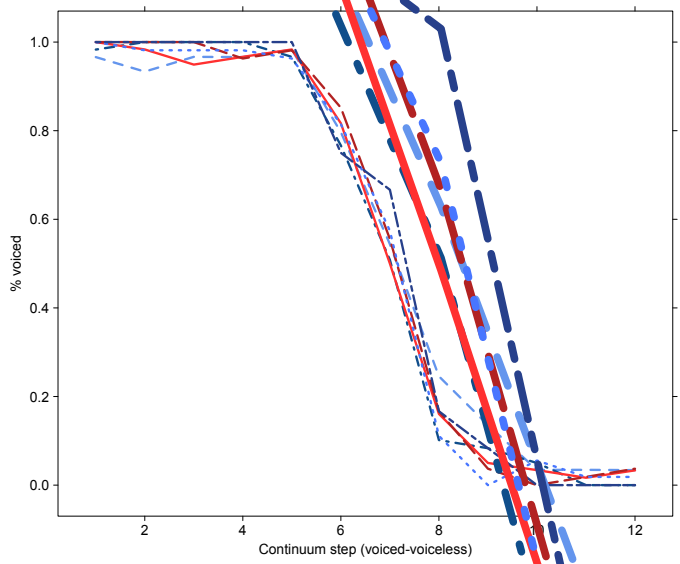

\section{Learner group}

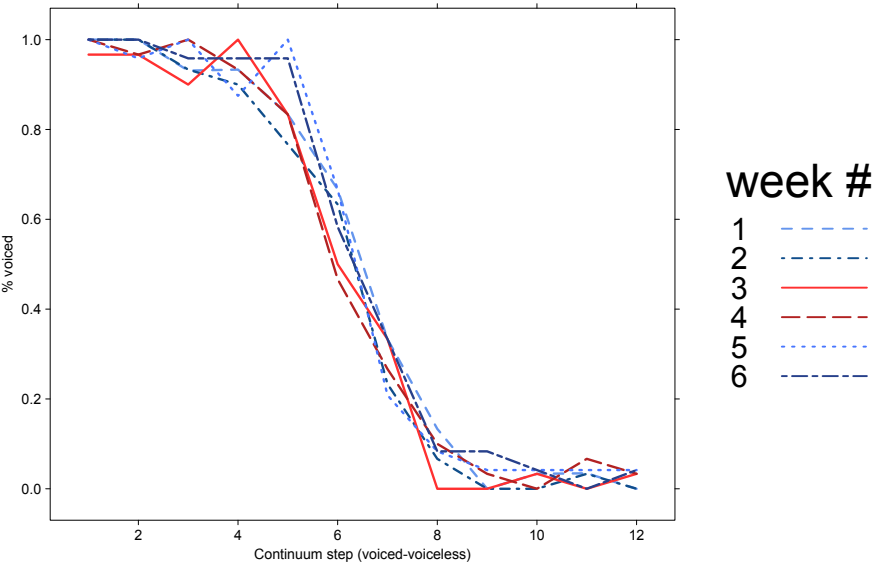

Figure 1. Phoneme categorization task results for Control (left) and Learner (right) participants over the 6-week testing period. Learners show more overall uncertainty, a lower average crossover point, and a slight shift downward in voiced-voiceless VOT boundary during weeks 3-4.

The semantic priming task showed a shift in responses consistent with the voicedvoiceless boundary shift in the phoneme categorization task. At the first test (week 2), the learners showed strong priming for canonical P-type and B-type tokens (at 0 and $80 \mathrm{~ms}$ ) and approximately equal priming for ambiguous tokens of both types (at $20 \mathrm{~ms}$ ). But at the second test (week 4) B-type primes with 20 ms VOTs become less effective than 20 ms P-type primes, and 0 ms P-type primes actually inhibited recognition of their associated targets. This suggests that, by week 4, short (0 - $20 \mathrm{~ms})$ VOT values were not acceptable for English/b/-initial words.

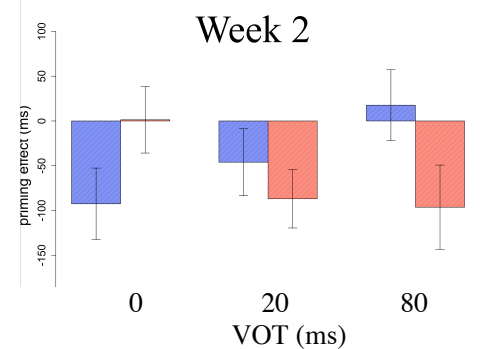

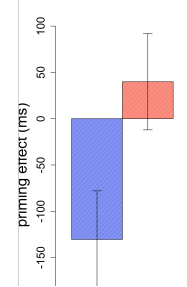

0
Week 4

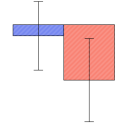

20
$\operatorname{VOT}(\mathrm{ms})$

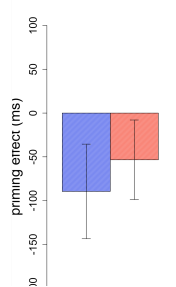

0
Week 6

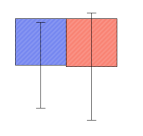

80

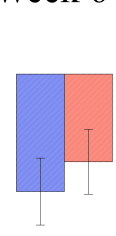

20
$\operatorname{VOT}(\mathrm{ms})$

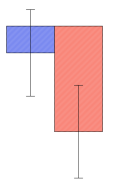

80

Figure 2. Semantic priming task results for the Learner group. Lower bars indicate a greater priming effect. Ambiguous tokens became better/p/ primes at week 4, consistent with the category boundary shift in Figure 1 .

At the third test (week 6) priming effects were greater across the board, suggesting that participants may have begun to exploit lexical cues other than word-initial VOT since the cue is not only unreliable in this task, but also as an indicator of the English voiced-voiceless distinction in their recent experience. No qualitative shifts were shown for the Control group.

Though the number of participants in our study is limited, we believe that these shifts demonstrate support for the notion that phonetic drift in novice L2 learners appears in L1 speech perception. During the first two weeks, participants appeared to simply behave as native monolingual English speakers would (i.e., our Control group), applying their L1 categories to tasks with French VOT. At weeks 3 and 4, however, they indicated drift-like behavior in their English processing across both tasks, suggesting some amount of L1 category confusion as a result of their two weeks of L2 input. At weeks 5 and 6 participants demonstrated a return to early behavior in the phoneme categorization task, but not in the semantic priming task. Here 
instead, we found that learners seemed to opt for a different strategy: using other lexical cues to identify items. Since no changes were found for either task in the Control group, we attribute the shifts in the Learner group to the learning environment in which they were immersed.

\section{Individual differences}

Each of the five participants in the Learner group showed these shifts in perception to some extent, but some more than others. We expect that variance across participants will be related to some of the other factors for which we have data: working memory, cognitive control, socio-motivational characteristics of each participant, daily usage of French, et cetera.

\section{Conclusions}

Novice L2 French learners showed perceptual shifts in their L1 English across two tasks in this longitudinal study of L1 speech perception, corroborating Chang's (2010) findings for production. As in production, L2 exposure influences L1 speech perception processes within weeks of language exposure. Whatever perceptual model we use to account for these changes will have to allow for complex adaptive behavior in which perception drifts and then reverts to L1 norms for more metalinguistic tasks, like phoneme categorization, but continues to evolve in a compensatory way for tasks that require higher-level linguistic processing.

Our future analyses will explore the link between each participant's perceptual shifts and any VOT-related shifting that arises in their L1 speech production. Since we have detailed information concerning each participant's non-linguistic cognitive skills and socio-motivational orientation toward learning French, we may be able to uncover what factors (besides age of acquisition) contribute to variation in second language learning. To investigate whether these effects are due to immersion itself or simply the intensive coursework for each participant, we will collect data from a second group of novice L2 French learners taking their classes in the United States. We hope that this project will give some insight into the links between individual differences, language learning, and speech processing.

\section{References cited}

Andruski, J. E., Blumstein, S. E. \& Burton, M. (1994). The effect of subphonetic differences on lexical access. Cognition 52, 163-187.

Bradlow, A. R., Akahane-Yamada, R., Pisoni, D. B. \& Tohkura, Y. (1999). Training Japanese listeners to identify English /r/ and /1/: Long-term retention of learning in perception and production. Perception and Psychophysics 61, 977-985.

Chang, C. B. (2010). First language phonetic drift during second language acquisition. Doctoral Dissertation. Manuscript, University of California, Berkeley.

Flege, J. E. (1987). The production of "new" and "similar" phones in a foreign language: Evidence for the effect of equivalence classification. Journal of Phonetics 15, 47-65.

Flege, J. E., Schirru, C. \& MacKay, I. R. A. (2003). Interaction between the native and second language phonetic subsystems. Speech Communication 40, 467-491.

Laeufer, C. (1996). Towards a typology of bilingual phonological systems. In A. James and J. Leather (Eds.), Second-Language Speech: Structure and Process (pp. 325-342). Berlin, Germany: Mouton de Gruyter. 